\title{
Microanalysis of Ferromagnetic-Ferroelectric Interfaces Using Analytical Electron Microscopy
}

\author{
P.S.Sankara Rama Krishnan ${ }^{*}$, Yang, Jan-Chi ${ }^{* *}$ Chu, Ying-Hao ${ }^{* *}$, Nagarajan Valanoor ${ }^{*}$ and \\ Paul Munroe*
}

* School of Materials Science \& Engineering, University of New South Wales, Sydney, NSW 2052, Australia

** Department of Materials Science and Engineering, National Chiao Tung University, Hsinchu, Taiwan.

Magnetic tunnel junction-based magnetic memory devices have became commercially very attractive because of their ability to simultaneously achieve high density, non-volatility and high speed[1]. A typical magnetic tunnel junction consists of two ferromagnetic electrodes separated by a thin $(\sim 5 \mathrm{~nm})$ insulating barrier. The electron tunneling across the barrier typically depends on the density of states at the electrode-barrier interface. Ferroelectric materials can also be used as a barrier instead of insulating oxides. For the tunnel junction architecture to perform effectively, the stability of ferroelectricity at ultra low dimensions is a very critical factor. The confirmation of existence of ferroelectricity at few unit cell dimensions[2] by both experimental and theoretical[3] studies has further enhanced the research potential for ferroelectric-based tunnel junction devices. The electric field induced polarization switching in ferroelectric materials can alter the polarity at the ferroelectric barrier-electrode interfaces [4]. Due to this additional functionality the barrier becomes an active component[5] in the tunnel junction heterostructure architecture. Thus, interfacial phenomena, such as chemical inhomogeneity and degree of strain can have a direct influence on the performance of such devices.

In this study, a ferromagnetic-ferroelectric-ferromagnetic heterostructure was fabricated on a strontium titanate (STO - 001) oriented substrate using pulsed laser deposition (PLD). STO has a lattice constant of $0.390 \mathrm{~nm}$. Lanthanum strontium manganese oxide (LSMO) with a lattice constant of $0.387 \mathrm{~nm}$ was used as a ferromagnetic electrode. Multiferroic bismuth ferrite (BFO) with a lattice constant of $0.396 \mathrm{~nm}$, served as a barrier. The ferromagnetic and multiferroic materials are selected in a way that there was a minimal difference in their lattice constant so that the lattice strain was minimised. In addition to this, using multiferroic (simultaneously display ferroelectric and ferromagnetic ordering) BFO provides additional functionality to the tunnel junction device architecture. In this structure, first the bottom LSMO layer was deposited at $700^{\circ} \mathrm{C}$, vacuum of 100 mTorr oxygen. This was followed by BFO layer. The top LSMO layer was deposited as a final step.

Two samples with different thicknesses of BFO (specifically either $5 \mathrm{~nm}$ or $20 \mathrm{~nm}$ ) were fabricated and the structure of their interfaces were characterised using transmission electron microscope (TEM) - bright field imaging and scanning transmission electron microscopeenergy dispersive spectroscopy (STEM-EDS). Cross-sectional TEM samples were prepared using a dual beam focused ion beam microscope (Nova 200, FEI Co.). A Philips CM 200 
(FEG) operated at $200 \mathrm{kV}$ with a probe size of $\sim 1.5 \mathrm{~nm}$ was used for imaging and STEMEDS analysis. The acronyms used to describe the samples were LS for LSMO, BF for BFO followed by each layer thickness.

Bright field TEM images of LS80BF5LS80 and LS90BF20LS90 are shown in figures $1 \mathrm{a}$ and b. It can be observed that there are no visible dislocations or other crystalline defects at either the inner (LSMO-BFO) or outer (BFO-LSMO) interfaces. Both interfaces were able to maintain the physical integrity every at ultra low $(\sim 5 \mathrm{~nm})$ dimensions and at higher thickness $(\sim 20 \mathrm{~nm})$ levels.

Elemental maps, acquired via STEM-EDS, of LS80BF20LS90 are shown in figure 2. The STEM-EDS chemical mapping for elements Sr-K $\alpha, \mathrm{Mn}-\mathrm{K} \alpha, \mathrm{Bi}-\mathrm{L} \alpha$ and $\mathrm{Fe}-\mathrm{K} \alpha$ was carried out using windows located at peak energies of 14.16, 5.89, 10.83, and $6.40 \mathrm{keV}$, respectively, with an energy window width of $0.2 \mathrm{keV}$ at these energies. The images show distinct contrast for each element at their respective layers, indicating, up to the levels of resolution, that the layers are chemically free from any diffusion. Similar trend was observed in LS80BF5LS90 sample as well (not reported here). The dissociation of BFO into parasitic[6] $\mathrm{Fe}_{2} \mathrm{O}_{3}$ and $\mathrm{BiO}_{2}$ at temperatures close to PLD deposition conditions and the diffusion [7] of Fe into LSMO without disturbing the physical stability of the interface is a great concern for performance of BFO based devices. In this present study the chemical and physical stability of the BFO interface was achieved which can make the ferroelectric tunnel junction architecture performance more reliable.

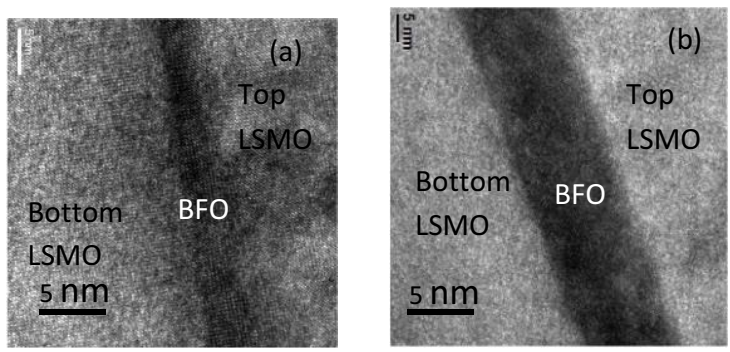

Figure $1 \mathrm{a}-1 \mathrm{~b}$. Bright field TEM images of the LSMO-BFO-LSMO heterostructures
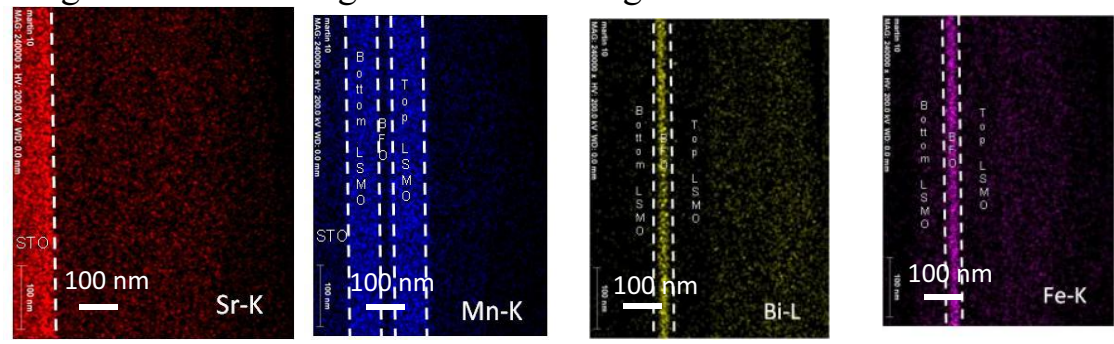

Figure 2. STEM-EDS chemical mapping of Sr-K, Mn-K, Bi-L, Fe-K elements for LS80BF20LS90 sample References:-

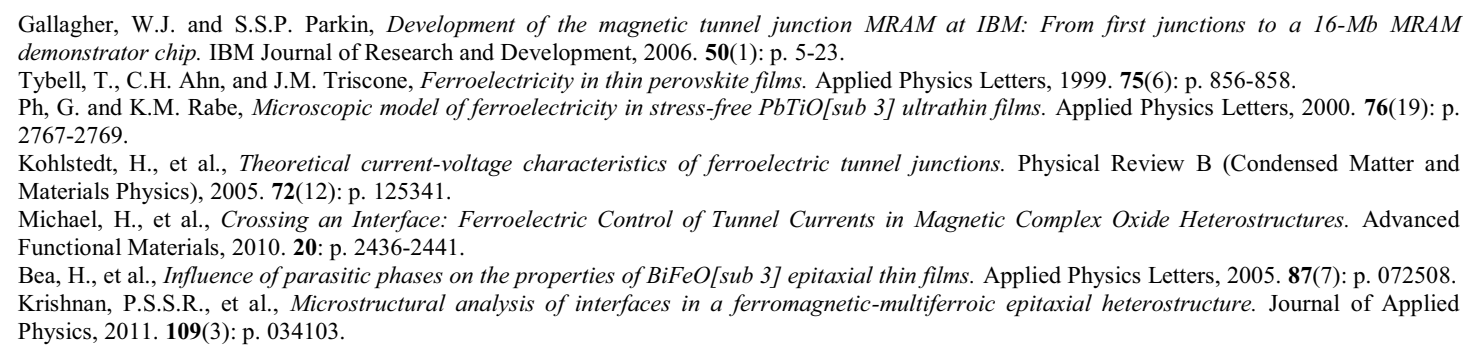

